\title{
Bubble PIV technique to measure the velocity field of a free-swimming California sea lion
}

\author{
Gino Perrotta ${ }^{1}$, Frank E. Fish ${ }^{2}$, Megan C. Leftwich ${ }^{1 *}$ \\ ${ }^{1}$ George Washington University, Department of Mechanical and Aerospace Engineering, Washington, \\ USA \\ ${ }^{2}$ West Chester University, 2Department of Biology, West Chester, USA \\ *mleftwich@gwu.edu
}

\section{Introduction}

Fish et al. (2014) adapted laboratory PIV for safe use on larger animals. As opposed to seeding the entire flow with reflective particles and illuminating a plane of the flow with a laser, they produced a sheet of small bubbles and used sunlight for global illumination. Underwater cameras imaged the flow in a method similar to traditional PIV. This technique was used to measure the flow around a swimming dolphin and estimate the thrust produced during a tail stand maneuver (Fish et al. (2014, 2018)). In the current work, we will extend the modification of PIV of Fish et al. to measure the flow produced by a swimming sea lion also using bubbles as seeding particles and sunlight as illumination. This is the first time that the flowfield of a swimming sea lion has been directly measured. We will present an extensive extension to the image processing required to measure flow under field conditions. Finally, we will present the flow generated by propulsive strokes of an adult female (Cali) sea lion freely swimming through a pool of stationary water.

\section{Methods}

Data collection: Measurements were taken at Science Learning and Exploration With The Help of Sea lions (SLEWTHS) in Moss Landing, California. Three adult California Sea Lions trained to follow an instructor?s target were guided to swim through a sheet of bubbles in front of a submerged camera. Small bubbles were generated in a thin flat sheet by releasing compressed air through a porous irrigation hose placed on the floor of the pool. The motion of the bubbles was recorded by a submerged GoPro Hero 5 at 1920x1080 pixels and 120 fps. Image processing: The raw images need extensive processing to yield clear velocity fileds. The process, and results are shown in Fig. 1. The process involves xxx steps: image data reduction (Fig. 1(b)), a sliding minimum subtraction (Fig. 1(c)), a spatial median subtraction (Fig. 1(d)), a heuristic refinement (Fig. 1(e)), a two-dimensional PIV calculation (Fig. 1(f)), and a three-dimensional median subtraction (Fig. $1(\mathrm{~g}))$. This lengthy process is necessary largely due to the use of global illumination via sunlight and the resulting reflections throughout the large field of view.

\section{Results}

The flowfield measured in the bubble-plane was measured for 173 runs of three separate individuals. Fig. $1(\mathrm{~g})$ shows the velocity magnitude in a representative run. Because the animals swim freely in the pool, ensuring in-plane motion and propulsive stroke timing necessitate a large number of runs, a feat made more challenging by the difficulties of working with marine mammals. From these data we are able to examine the full-scale flowfield produced by the swimming sea lion, and estimate the strength of the resulting fluid jet. Overall, XXX flowfields were measured showing distinct flow features created by the sea lion at various points in the propulsive stroke. The method was repeated in a water channel at West Chester University at known steady velocity. This allowed for validation of the technique by measuring a known flowfield in a controlled setting (something this is not possible in the field experiments). 

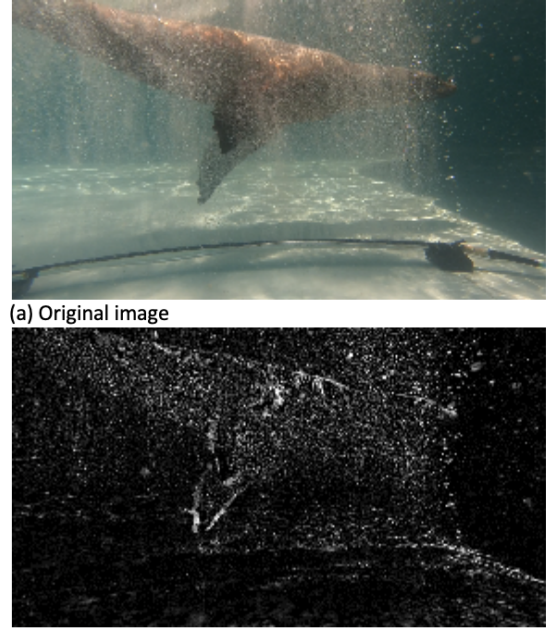

(c) Temporal minimum subtraction

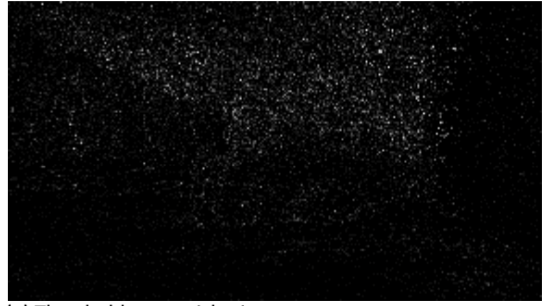

(e) Threshold on particle size

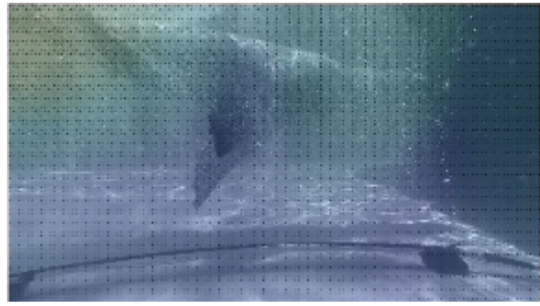

(g) 3D median filter
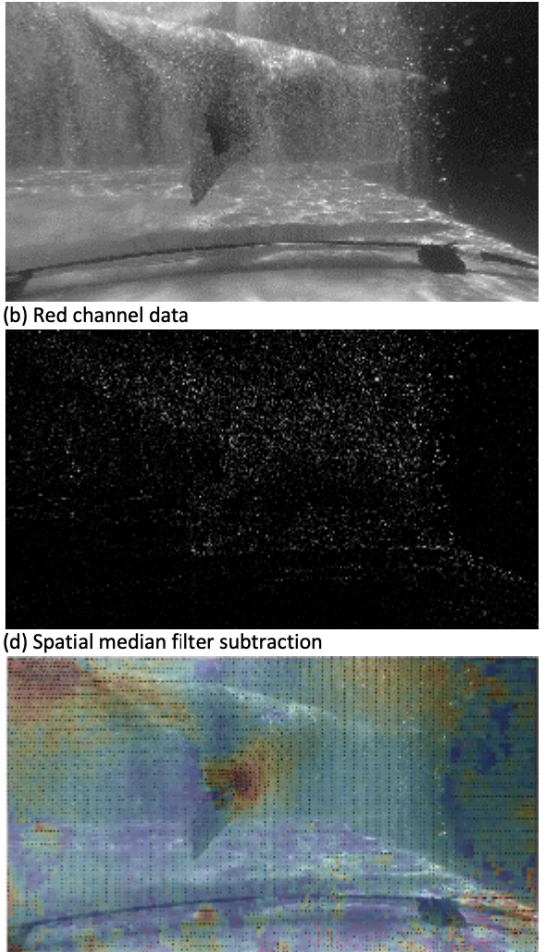

(f) 2D PIV (in DaVis)

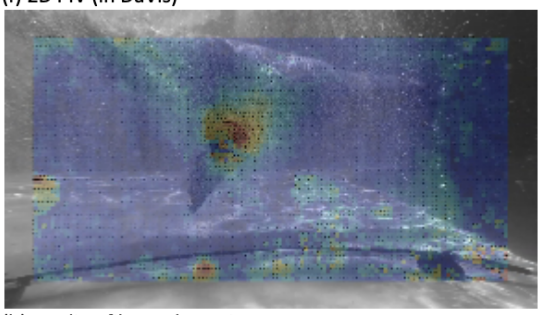

(h) Median filter subtraction

Figure 1: Each panel shows a step in the data processing from the raw data (a) to the measured flowfield (h).

\section{Conclusions}

The flowfield of swimming California sea lions was measured by adapting and advancing bubble PIV techniques. These results not only illuminate this particular flow phenomenon, they also provide additional options for measuring flowfields in field settings where typical PIV techniques are not feasible.

\section{Acknowledgements}

Finding for this study was provided by the Office of Naval Research Award N000141712312 (PO Tomas McKenna) to MCL and FEF and Award N000141712448 (PO Robbert Brizzolera) to MCL. As well as the National Science Foundation Award CBET 1604876 to MCL.

\section{References}

Fish FE, Legac P, Williams TM, and Wei T (2014) Measurement of hydrodynamic force generation by swimming dolphins using bubble dpiv. Journal of Experimental Biology 217:252-260

Fish FE, Williams TM, Sherman E, Moon YE, Wu V, and Wei T (2018) Experimental measurement of dolphin thrust generated during a tail stand using dpiv. Fluids 3:33 